circulating levels of CTSS after adjustment for the Global Registry of Acute Coronary Events (GRACE) score, which is widely recommended for risk stratification in non-ST-segment elevation acute coronary syndrome (NSTE-ACS).

Methods CTSS was measured in blood samples collected from 1,129 consecutive patients with adjudicated NSTE-ACS presenting at an acute chest pain unit for evaluation of a possible acute coronary syndrome. Cardiovascular (CV) death and a composite of all-cause mortality and AMI were evaluated as the primary and secondary endpoints of the study, respectively. The additive prognostic value of CTSS over the GRACE score was estimated by the Net Reclassification Index (NRI) that examines the net upward and downward reclassification into correct pre-defined risk categories.

Results After a median follow-up of 21 months, 101 (8.95\%) deaths were reported, of which 63 (5.6\%) were of cardiac origin. The combined endpoint occurred in 176 (15.6\%) patients. Patients with CTSS in the highest tertile presented the greatest risk for all-cause mortality $(\mathrm{HR}=1.84$ for highest versus lowest tertile of CTSS distribution, 95\% CI 1.1-3.08, $\mathrm{P}=0.02)$ and $\mathrm{CV}$ death $(\mathrm{HR}=2.5$ for highest versus lowest tertile of CTSS distribution, 95\% CI 1.24-5.05, $\mathrm{P}=0.011$ ) after adjustment for age, gender, diabetes mellitus, hs-cTnT, hsCRP, revascularization and index diagnosis. Similarly, CTSS was associated with increased risk of cardiovascular death after adjusting for the GRACE Score (adjusted HR for highest versus lowest tertile of CTSS distribution $=2.34$, 95\% CI 1.18 4.64, $\mathrm{P}=0.015)$. Further, CTSS predicted the combined endpoint of all-cause death or non-fatal MI independently of the GRACE Score (adjusted HR for highest versus lowest tertile of CTSS distribution=1.67, 95\% CI 1.15-2.42, $\mathrm{P}=0.007$ ). When CTSS was added over the GRACE Score, it conferred significant reclassification value for CV death (NRI $=21.4 \%$, $\mathrm{P}=0.008$ ). Similarly, CTSS correctly reclassified risk for allcause death or non-fatal MI $(\mathrm{P}=0.006)$ in $15.9 \%$ of the population.

Conclusion Circulating CTSS predicts mortality and improves risk stratification of patients with NSTE-ACS over the GRACE score recommended by clinical guidelines. The clinical application of CTSS as a novel biomarker in NSTE-ACS should be further explored and validated.

Conflict of Interest none

\section{BEST-HEART SCORE: A RE-EVALUATION OF THE HEART SCORE IN THE ERA OF POINT OF CARE TROPONIN TESTING}

${ }^{1}$ Garry McDowell ${ }^{*},{ }^{1}$ Malak Almashali, ${ }^{2}$ Niall Morris, ${ }^{2}$ Richard Body. ${ }^{1}$ Manchester Metropolitan University; ${ }^{2}$ University of Manchester; ${ }^{4}$ University of Manchester
Introduction Point of care test has many advantages including access and time to result. The major disadvantage in POC for cardiac troponin is the lack of high-sensitivity assays, matching the analytical performance of central laboratory testing. The HEART score can easily be applied in the Emergency Department using data collected as part of routine evaluation of the patient. The objective of this study, was to re-evaluate the HEART score, using a score of $0-2$ as low risk, accounting for the poorer analytical sensitivity of POC troponin assays.

Methods This is a sub-study of the Bedside Evaluation of Troponin (BEST) Study. We recruited patients presenting to the ED with suspected Acute Coronary Syndromes. Blood for POC TnI (LoD $20 \mathrm{ng} / \mathrm{L}$; 99th Percentile $80 \mathrm{ng} / \mathrm{L}$ and Functional Sensitivity, $\mathrm{CV}<10 \%, 100 \mathrm{ng} / \mathrm{L})$ was drawn at presentation and 3 hours later. The HEART Score was calculated as published using the LoD, 99th percentile and functional sensitivity as the upper limit of normal for troponin. A HEART score of $0-2$ was taken as low risk (BESTHEART score). All patients underwent central laboratory testing over 3-12 hours. We also combined the BESTHEART score with a normal troponin at baseline and 3 hours using $20 \mathrm{ng} / \mathrm{L}, 80 \mathrm{ng} / \mathrm{L}$ and $100 \mathrm{ng} / \mathrm{L}$ as the ULN. The primary outcome was a diagnosis of ACS, defined as acute myocardial infarction during the initial presentation or major adverse cardiac event (MACE) within 30 days, including all-cause mortality, incident $\mathrm{MI}$ and coronary revascularisation. The diagnosis of AMI was based on the Third Universal Definition (table 1).

Results 614 patients (238 (38.2\%) female and 385 (61.8\%) male were included. ACS was reported in 86 (13.8\%). The AUC for the HEART score was $0.75(100 \mathrm{ng} / \mathrm{L}) ; 0.80(80 \mathrm{ng} / \mathrm{L})$ and $0.84(20 \mathrm{ng} / \mathrm{L})$, although compared to functional sensitivity this did not reach statistical significance $(p=0.97 \quad \& \quad 0.17$ respectively) (figure 1). A low risk BEST-HEART score using $20 \mathrm{ng} / \mathrm{L}$ (LoD) as the ULN had the greatest sensitivity (96.5\%) and NPV (98.5\%). Using the BEST-HEART score alone would have a probability of missed ACS of $1.5 \%$. Combining a low risk BEST- HEART score with a normal troponin (ULN 20 $\mathrm{ng} / \mathrm{L})$ over 3 hours improved the sensitivity (100\%) and NPV (100\%), ruling out $164(30.1 \%)$ patients.

Conclusion The BEST-HEART score had improved diagnostic accuracy compared to the traditional HEART score using a contemporary POC troponin assay. While the BEST-HEART score has insufficient sensitivity to exclude ACS, it may be used where access to central laboratory testing is not widely available if clinicians will accept the small risk of missed ACS. The addition of normal troponin over 3 hours improves the sensitivity and NPV, but requires an additional delay for follow up testing. This protocol should be prospectively validated prior to clinical use.

Conflict of Interest None 